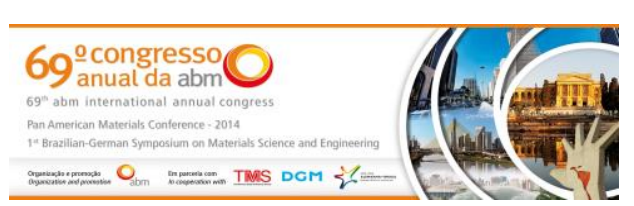

Tema: Corrosão

\title{
ESTUDO DO GRAU DE SENSITIZAÇÃO DO AÇO INOXIDÁVEL UNS S31803 ENVELHECIDO A $750^{\circ} \mathrm{C}^{*}$
}

\begin{abstract}
Luara da Costa Morais ${ }^{1}$ Daniella Caluscio dos Santos ${ }^{2}$

\section{Resumo}

O objetivo deste trabalho compreende o estudo do grau de sensitização (DOS) do aço inoxidável dúplex (AID) UNS 531803 envelhecido a $750^{\circ} \mathrm{C}$ por ensaios de DL-EPR. Para isto, corpos de prova do aço em estudo foram solubilizados a $1.175^{\circ} \mathrm{C}$ e envelhecidos a $750^{\circ} \mathrm{C}$ entre $10 \mathrm{~min}$ e $240 \mathrm{~h}$ visando a formação de diferentes frações de sigma. Corpos-de-prova metalográficos foram utilizados para a caracterização microestrutural, que foi realizada por meio de técnicas de estereologia quantitativa a partir de imagens de elétrons retroespalhados para a quantificação das fases intermetálicas formadas durante 0 envelhecimento e análises magnéticas. Os ensaios de DL-EPR foram conduzidos em solução $2 \mathrm{M}$ $\mathrm{H}_{2} \mathrm{SO}_{4}+0,5 \mathrm{M} \mathrm{NaCl}+0,01 \mathrm{M} \mathrm{KSCN}$ à $30 \pm 2^{\circ} \mathrm{C}$, em amostras polidas. Observou-se que o DOS das amostras envelhecidas mostra forte dependência da microestrutura. Foram registrados duplos máximos de densidade de corrente tanto na ativação quanto na reativação, onde os ensaios potenciostáticos mostraram que estes máximos estão relacionados a dissolução da fases, ferrita e austenita. Observou-se o aumento no DOS de ambas as fases seguido de queda, provavelmente devido à redistribuição de $\mathrm{Cr}$ para regiões empobrecidas, decorrentes da formação de fases intermetálicas.
\end{abstract}

Palavras-chave: Aço inoxidável dúplex; Fase sigma; Grau de sensitização; DL-EPR.

\section{STUDY OF THE DEGREE SENSITIZATION IN UNS S31803 STEEL BY DOUBLE LOOP POTENTIODYNAMIC REACTIVATION TESTS}

\section{Abstract}

The main purpose of this research is the study of the degree of sensitization (DOS) of UNS S31803 duplex stainless steel (DSS) aged at $750^{\circ} \mathrm{C}$ by DL-EPR tests. For this, the as received material was solution treated at $1,175^{\circ} \mathrm{C}$ for 30 minutes and aged at $750^{\circ} \mathrm{C}$ between $10 \mathrm{~min}$ and $240 \mathrm{~h}$, enabling the formation of different $\sigma$ phase contents. The aged samples were mounted in metallography specimens for microstructural characterization that was carried out by quantitative metallography, magnetic measurements and scanning electron microscopy (SEM). The DL-EPR tests were conducted in polished samples at $30^{\circ} \mathrm{C}$ in a $2 \mathrm{M} \mathrm{H}_{2} \mathrm{SO}_{4}+0,5 \mathrm{M} \mathrm{NaCl}+$ $0,01 \mathrm{M}$ KSCN solution. It was registered a double maximum current density both in activation and reactivation processes. Potenciostatic tests confirms that the current maximum detected are relates to show that this maximum are related the dissolution of ferrite and austenite phases. It was also discussed that the increase followed by slight decrease of the DOS values for both phases are probably related by $\mathrm{Cr}$ redistribution to depleted zones generated by intermetallic phases formation.

Keywords: Duplex stainless steel; Sigma phase; Degree of sensitization; DL - EPR.

1 Engenheira de Materiais, Centro Universitário da FEl, São Paulo, Brasil; luaracmorais@yahoo.com.br.

2 Engenheira química, Profä. Drạ., Departamento de Engenharia de Materiais, Centro Universitário da FEl, São Paulo, Brasil; dcaluscio@fei.edu.br.

* Contribuição técnica ao 69 Congresso Anual da ABM - Internacional e ao 14ํㅡㄹ ENEMET - Encontro Nacional de Estudantes de Engenharia Metalúrgica, de Materiais e de Minas, 21 a 25 de julho de 2014, São Paulo, SP, Brasil. 


\section{INTRODUÇÃO}

Da necessidade de um aço que combinasse boas propriedades mecânicas e boa resistência a corrosão, foi desenvolvido um aço bifásico, com estrutura aproximada de $50 \%$ ferrita $(\alpha)$ e $50 \%$ austenita $(\gamma)$, denominado aços inoxidável duplex (AID). A aplicação desses aços se dá principalmente em indústrias químicas, óleo e gás, poços, sistemas de incêndios, equipamentos para controle de poluição, indústria de papel e celulose, dentre outras aplicações [1-3].

Contudo, os aços dúplex quando expostos a temperaturas entre $650^{\circ} \mathrm{C}$ e $950^{\circ} \mathrm{C}$ podem formar fases intermetálicas. As principais fases passíveis de formação nestes aços são sigma $(\sigma)$, chi $(\chi)$, e nitretos principalmente do tipo $\mathrm{Cr}_{2} \mathrm{~N}$ [4-6]. Segundo Nilsson [6] dentre estes precipitados a fase sigma é a mais importante, pois por ser rica em $\mathrm{Cr}$ e Mo causa o empobrecimento destes elementos de liga nas regiões adjacentes a sua formação exercendo grande influência no comportamento eletroquímico dos aços inoxidáveis dúplex.

Segundo Nilsson [6] e Magnabosco [7] nos aços AID a fase $\sigma$ pode ser formar por três mecanismos: (a) nucleação e crescimento a partir da $\alpha$, resultado na formação de ferrita secundária $\left(\alpha_{2}\right)$, pobre em $\mathrm{Cr}$ e Mo que dependendo de seu empobrecimento em $\mathrm{Cr}$, e enriquecimento em $\mathrm{Ni}$ poderia se transformar em austenita secundária $\left(\gamma_{2}\right)$; (b) decomposição eutetóide da $\alpha$ gerando $\gamma_{2}$ pobre em Cr e Mo; (c) crescimento a partir da $\gamma$ após total consumo de ferrita, formando austenita secundária pobre em Cr e Mo.

A formação de regiões empobrecidas em $\mathrm{Cr}$ e Mo tem forte influência na resistência à corrosão localizada [7], e estudos recentes analisam essa influência utilizando técnicas de reativação potenciodinâmica de duplo-loop (DL-EPR), que foi desenvolvido incialmente para os aços inoxidáveis austeníticos, mas que hoje tem sido aplicado aos AID [8-17].

Chavez e Wolynec [12] avaliando o comportamento eletroquímico do aço UNS S31803 envelhecido a $750{ }^{\circ} \mathrm{C}$ com ensaios de DL-EPR em solução $2 \mathrm{M} \mathrm{H}_{2} \mathrm{SO}_{4}$ $+0,5 \mathrm{M} \mathrm{NaCl}+0,01 \mathrm{KSCN}$ reportaram a presença de duplos máximos de densidade de corrente na reativação. Segundo os autores o máximo de maior potencial se refere à dissolução de regiões empobrecidas pela formação de sigma e o de menor potencial à dissolução de regiões empobrecidas pela formação de carbonetos, chi e nitretos. Cristini et al [13] sob as mesmas condições de ensaio e com o mesmo material utilizado por Chavez e Wolynec [12], também reportaram a presença de duplos máximos de densidade de corrente, entretanto, segundo os autores os máximos encontrados referem-se a dissolução das fases, ferrita e austenita, sendo o de menor potencial relacionado a dissolução da primeira e o de maior a segunda. A afirmação de Cristini et al. [13] também é sugerida por Morais, Santos e Magnabosco [10] que observaram a presença de duplos máximos de densidade de corrente no aço UNS S31803 onde o máximo de menor potencial atenua-se com o aumento do tempo de envelhecimento, paralelamente à diminuição da fração de ferrita.

Estudos que avaliam o grau de sensitização nos AID reportam oscilações na variação do grau de sensitização com o tempo de envelhecimento [9,11-17]. Segundo Gao et al. [17] estas oscilações podem estar relacionadas a redistribuição de $\mathrm{Cr}$ e Mo de regiões menos empobrecidas para as mais empobrecidas devido a formação de fases intermetálicas.

Diante do exposto, nota-se que o comportamento eletroquímico sob ensaio DL-EPR dos aços inoxidáveis duplex possui forte dependência da microestrutura e das fases

* Contribuição técnica ao 69ำ Congresso Anual da ABM - Internacional e ao 14ํㅡㄹ ENEMET - Encontro Nacional de Estudantes de Engenharia Metalúrgica, de Materiais e de Minas, 21 a 25 de julho de 2014, São Paulo, SP, Brasil. 


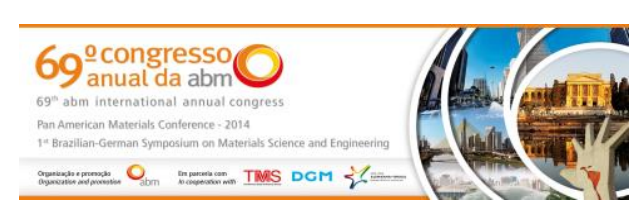

intermetálicas passíveis de formação nesses aços. Assim, o presente trabalho procura estudar o grau de sensitização do aço UNS S31803 envelhecido a $750^{\circ} \mathrm{C}$ em solução $2 \mathrm{M} \mathrm{H}_{2} \mathrm{SO}_{4}+0,5 \mathrm{M} \mathrm{NaCl}+0,01 \mathrm{M} \mathrm{KSCN}$ por ensaios DL-EPR.

\section{MATERIAL E MÉTODOS}

O material em estudo foi adquirido como chapa de $3 \mathrm{~mm}$ de espessura, laminada a $1100^{\circ} \mathrm{C}$ e resfriada por jato de ar e água forçado. A composição química do material é apresentada na Tabela 1.

Tabela 1. Composição química (\% em massa) do aço UNS S31803 em estudo

\begin{tabular}{ccccccccc}
\hline $\mathrm{Cr}$ & $\mathrm{Ni}$ & $\mathrm{Mo}$ & $\mathrm{Mn}$ & $\mathrm{N}$ & $\mathrm{C}$ & $\mathrm{Si}$ & $\mathrm{Cu}$ & $\mathrm{Fe}$ \\
\hline 22,48 & 5,74 & 3,20 & 1,42 & 0,162 & 0,018 & 0,35 & 0,15 & Bal. \\
\hline
\end{tabular}

A partir do material como recebido, foi realizado tratamento térmico de solubilização a $1.175^{\circ} \mathrm{C}$ por $30 \mathrm{~min}$, seguido de resfriamento em água buscando-se estrutura homogênea de $\alpha$ e $\gamma$. Em seguida amostras foram envelhecidas por tempos entre $10 \mathrm{~min}$ e $240 \mathrm{~h}$ a $750^{\circ} \mathrm{C}$, o envelhecimento foi realizado em forno tubular sob atmosfera de $\mathrm{N}_{2}$ para se evitar oxidação excessiva, seguido de resfriamento em água.

As amostras envelhecidas tiveram todas as suas faces lixadas até a obtenção de superfície com o acabamento conferido por lixa de granulometria 100 mesh, em seguida foram embutidas em resina fenólica de cura a quente (baquelite), gerando corpos-de-prova metalográficos que foram lixados até granulometria de 600 mesh, e em seguida foram polidos até $1 \mu \mathrm{m}$. A superfície de observação dos corpos-de-prova corresponde à seção longitudinal da chapa, com relação à direção de laminação, com área exposta variando entre 0,4 e $0,6 \mathrm{~cm}^{2}$.

A quantificação da $\alpha$ foi feita por análises magnéticas e a quantificação de $\sigma$ e $\chi$ por estereologia quantitativa a partir de imagens obtidas por elétrons retroespalhados. Já a fração de $\gamma$ foi calculada pelo balanço em relação à somatória das demais fases $\% \gamma=100-(\% \alpha+\% \sigma+\% \chi)$. As análises magnéticas foram realizadas com um ferritoscópio calibrado com o auxilio de padrões e tendo como limite de detecção $0,1 \%$ de $\alpha$. Vinte medições foram realizadas em cada uma das séries das amostras. Já a quantificação por imagens de elétrons retropalhados foi realizada em microscópio eletrônico CAMSCAN CS3200LV, de onde foram obtidas imagens das amostras polidas. Para determinação da fração volumétrica das fases $\chi \mathrm{e} \sigma$, foram obtidas 6 imagens de elétrons retroespalhados por amostra. Para que as amostras fossem obtidas de maneira satisfatória, o ajuste do microscópio eletrônico foi padronizados para todas as imagens: tensão de aceleração aplicada ao feixe de elétrons, mantida em $20 \mathrm{kV}$; ajuste da corrente elétrica emitida pelo filamento, mantida em $100 \mu \mathrm{A}$; diâmetro de feixe (spot size) constante; distância de trabalho, mantida em $20 \mathrm{~mm}$; e manutenção de valores constantes de brilho e contraste das imagens. Esses ajustes garantiram que as tonalidades e o delineamento das fases fossem os mesmos em todas as imagens coletadas, evitando diferenças na identificação das fases. A partir das imagens de elétrons retroespalhados a caracterização quantitativa da microestrutura foi realizada com o auxílio do software de análise de imagens Olympus AnalysisDoku.

Com o auxílio de potenciostato Autolab 12, foram realizados os ensaios DL-EPR utilizando solução $2 \mathrm{M} \mathrm{H}_{2} \mathrm{SO}_{4}+0,5 \mathrm{M} \mathrm{NaCl}+0,01 \mathrm{M} \mathrm{KSCN}$. As soluções foram preparadas com reagente padrão analítico e água destilada e deionizada. $\mathrm{O}$ ensaio foi conduzido a $30^{\circ} \mathrm{C}$, e foi utilizado o eletrodo de calomelano saturado (ECS) como

* Contribuição técnica ao 69 Congresso Anual da ABM - Internacional e ao 14ํㅡㄹ ENEMET - Encontro Nacional de Estudantes de Engenharia Metalúrgica, de Materiais e de Minas, 21 a 25 de julho de 2014, São Paulo, SP, Brasil. 
envelhecimento, em contrapartida nota-se nas curvas de DL-EPR a atenuação do máximo de menor potencial. Este comportamento também sugere que os duplos máximos de densidade de corrente podem estar relacionado a dissolução da $\alpha$ e $\gamma$.

A fim de avaliar o comportamento eletroquímico no material em cada máximo de densidade de corrente, foram realizados ensaios potenciostáticos na amostra envelhecida por $4 \mathrm{~h}$. A Figura 10a apresenta a imagem de elétrons secundários após o ensaio potenciostático no menor potencial $(-325 \mathrm{mV})$ onde se observa dissolução preferencial da $\alpha$. Na Figura 10b é apresentada a micrografia após o ensaio no maior potencial $(-225 \mathrm{mV})$, onde se observa o consumo da fase $\gamma$.

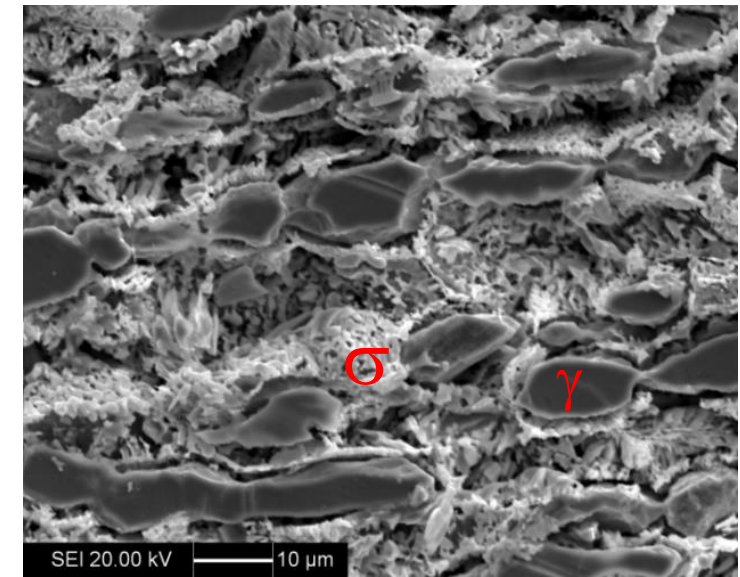

(a)

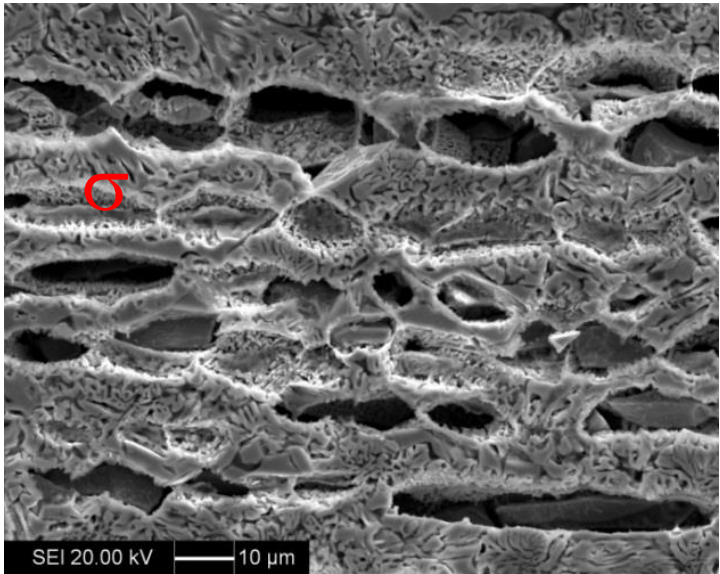

(b)

Figura 10. Imagem de elétrons secundários após ensaio potenciostático (a) $-325 \mathrm{mV}$ e (b) $-225 \mathrm{mV}$.

Desta maneira, verifica-se que o máximo de densidade de menor potencial é relacionado à dissolução da $\alpha$ e o de maior potencial à dissolução da $\gamma$, portanto os valores de $\mathrm{i}_{\mathrm{r} 1} / \mathrm{i}_{\mathrm{a} 1} \mathrm{e} \mathrm{i}_{\mathrm{r} 2} / \mathrm{i}_{\mathrm{a} 2}$ são relacionado respectivamente à $\alpha$ e a $\gamma$ e denominados DOS $_{\alpha}$ e DOS .

No estudo da variação do DOS com o tempo de envelhecimento (Figura 4) nota-se que tanto para a $\alpha$, quanto para a $\gamma$, até 40 min de envelhecimento, não há aumento significativo do DOS. Nesse trecho a fração de fases intermetálica formada é baixa, e o aumento de $\gamma$ e diminuição de $\alpha$ observados ao início do envelhecimento são provavelmente relacionados ao reequilíbrio entre $\alpha$ e $\gamma$ após a solubilização do material. Por isso provavelmente a formação de regiões empobrecidas que aumentaria o DOS é baixa.

Na Figura 4a é observado aumento no $\mathrm{DOS}_{\alpha}$ entre $40 \mathrm{~min}$ e $1 \mathrm{~h}$ de envelhecimento, provavelmente relacionado ao consumo de $\alpha$ decorrente da formação de fases intermetálicas $(\sigma+\chi)$ e consequentemente à formação de fases empobrecidas decorrentes da sua formação. Entretanto se observa na mesma figura diminuição no $D \mathrm{~S}_{\alpha}$ após $2 \mathrm{~h}$ de envelhecimento, provavelmente relacionada à redistribuição de $\mathrm{Cr}$ de regiões menos empobrecidas para as mais empobrecidas neste elemento, conforme sugerido por Gao et al. [17]. Além disso, vale ressaltar que após $2 \mathrm{~h}$ de envelhecimento há o consumo de chi, provavelmente relacionado à sua transformação em sigma, que provocaria nova redistribuição de $\mathrm{Cr}$ e Mo para regiões adjacentes à sua formação e que pode ter influenciado o comportamento observado.

Já no estudo do DOS em função do tempo de envelhecimento (Figura 4b) nota-se aumento no DOS até $72 \mathrm{~h}$ de envelhecimento. Entretanto, somente a partir desse tempo é observada leve queda nos valores de DOS $\mathrm{O}$. a aumento do DOS $\gamma$

* Contribuição técnica ao 69ำ Congresso Anual da ABM - Internacional e ao 14ํㅡㄹ ENEMET - Encontro Nacional de Estudantes de Engenharia Metalúrgica, de Materiais e de Minas, 21 a 25 de julho de 2014, São Paulo, SP, Brasil. 
duplo-loop (DL-EPR). In: Anais do 67 Congresso anual da ABM; 2012; Rio de Janeiro, Brasil. São Paulo: ABM; 2012.

10 Morais LC, Santos DC, Magnabosco R. Exploratory study of degree sensitization of duplex stainless steel UNS S31803 aged at $800^{\circ} \mathrm{C}$ by double loop potentiokinectic reactivation method (DL - EPR). In: Anais do European Corrosion Congress; 2013; Estoril, Portugal. Eurocorr; 2013.

11 Mélo EB, Magnabosco R, Moura-Neto C. Influence of the microstructure on the degree of sensitization of a duplex stainless steel UNS S31803 aged at $650^{\circ} \mathrm{C}$. Materials Research. 2014;16(6):1336-43.

12 Chaves R, Wolynec S. Investigação da corrosão seletiva do aço inoxidável dúplex UNS S31803 por meio de ensaios de reativação potenciocinética de ciclo duplo. In: 58응 Congresso Anual da ABM; 2003; Rio de Janeiro, Brasil. São Paulo: ABM; 2003.

13 Cristini SI, Sacchi B, Guerrini E, Trasatti S, Trasatti StP. Detection of sigma phase in $22 \% \mathrm{Cr}$ duplex stainless steels by electrochemical methods. Russian Journal of Electrochemistry. 2010;46(10):1168-75.

14 Ortiz N, Curiel FF, López VH, Ruiz A. Evaluation of the intergranular corrosion susceptibility of UNS S31803 duplex stainless steel with thermoeletric power measurements. Corrosion Science. 2013;69:236-244.

15 Lo KH, Kwok CT, Chan WK. Characterization of duplex stainless steel subjected to longterm annealing in the sigma phase formation temperature range by the DL-EPR test. Corrosion Science. Ano;53:3697-3703.

16 Amadou T, Bhaham C, Sidhom H. Double loop electrochemical potentiokinetic reactivation test optimization in checking of duplex stainless steel intergranular corrosion susceptibility. Metallurgical and Materials Transactions A. 2004;35A(11):3499-3515.

17 Gao J, Jiang Y, Deng B, Zhang W, Zhong C, Li J. Investigation of selective corrosion resistance of aged lean duplex stainless steel 2101 by non-destructive electrochemical techniques. Electrochimica Acta. 2009;54:5830-35.

18 Escriba DM, Materna-Morris E, Plaut RL, Padilha AF. Chi-phase precipitation in a dúplex stainless steel. Materials Characterization. 2009;60(11):1214-19.

19 Santos DC, Magnabosco R, Moura-Neto C. Influência do método de quantificação de fases na análise da formação de sigma em aço inoxidável duplex UNS S31803. In: Anais do 67ํㅡㄹ Congresso Anual da ABM; 2012; Rio de Janeiro, Brasil. São Paulo: ABM; 2012.

* Contribuição técnica ao 69 Congresso Anual da ABM - Internacional e ao 14ํㅡㄹ ENEMET - Encontro Nacional de Estudantes de Engenharia Metalúrgica, de Materiais e de Minas, 21 a 25 de julho de 2014, São Paulo, SP, Brasil. 